\title{
Institution of a Surgical Curriculum for Trabecular Microbypass Stent Placement
}

\author{
Farida Esaa Hakim, MD ${ }^{1}$ Varun Malhotra, MD ${ }^{2}$ Kathryn A. Colby, MD, PhD ${ }^{2} \quad$ Kamran M. Riaz, MD ${ }^{2}$ \\ ${ }^{1}$ Division of Biological Sciences, Pritzker School of Medicine, \\ University of Chicago, Chicago, Illinois \\ 2 Department of Ophthalmology and Visual Science, The University of \\ Chicago Medicine \& Biological Sciences, Chicago, Illinois \\ Address for correspondence Kamran M. Riaz, MD, Department of \\ Ophthalmology and Visual Science, The University of Chicago, 5841 \\ South Maryland Avenue, AMB S-437, Chicago, IL 60637 \\ (e-mail: kriaz@bsd.uchicago.edu). \\ J Acad Ophthalmol 2018;10:e143-e149.
}

\begin{abstract}
Keywords

- trabecular microbypass stent

- graduate medical education

- surgical education

- ophthalmology education

- surgical curriculum

- resident outcomes

Purpose This article evaluates whether a targeted, stepwise curriculum for microbypass stent placement leads to successful insertion of the iStent (Glaukos, Laguna Hills, CA) by resident surgeons.

Participants Senior (postgraduate year 4) ophthalmology residents (9 residents) from three classes during the 2015 to 2016, 2016 to 2017, and 2017 to 2018 academic years. Design Residents participated in a three-stage surgical curriculum. First, a wet laboratory was held for residents to gain familiarity with the device and develop bimanual surgical proficiency. The wet laboratory involved several stations, with each station requiring increased dexterity for successful completion. Next, residents practiced bimanual intraoperative gonioscopy after routine phacoemulsification procedures. Finally, residents performed combined phacoemulsification and iStent insertion under the supervision of an experienced attending surgeon. Primary success was determined by correct anatomical placement of the device confirmed via subsequent intraoperative gonioscopy by the supervising surgeon. Secondary success was measured by change in intraocular pressure (IOP) and number of topical hypotensive medications used after surgery. Residents provided feedback about the effectiveness of the curriculum via an online survey.

Results There were a total of 43 cases. There were no significant intraoperative complications, including hyphema. The iStent location was confirmed during both intraoperative and postoperative gonioscopy and was noted to be in appropriate position for the duration of the follow-up period (6-12 months) for all patients. Average preoperative IOP in our subjects was $17.5 \pm 4.0 \mathrm{~mm} \mathrm{Hg}$ and mean number of preoperative medications was $1.4 \pm 1.0$. At 6 months' postoperative, average IOP was $14.5 \pm 2.2 \mathrm{~mm} \mathrm{Hg}$ (13\% reduction from baseline [ $\pm 15 \%])$. At 12 months' postoperative, average IOP was $14.4 \pm 3.5 \mathrm{~mm} \mathrm{Hg}$, with a reduction of $14 \%( \pm 18 \%)$. The mean number of medications at follow-up was $1.2 \pm 1.1$. Mean postoperative IOP at 6 and 12 months was significantly lower compared with baseline $(p=0.0002, p=0.0001)$. Residents agreed that the curriculum prepared them to perform iStent insertion during residency and most residents felt prepared after residency.

Conclusion This stepwise surgical curriculum for trabecular microbypass stent placement leads to successful performance of the procedure by residents and is a useful model for other residency programs.
\end{abstract}

received

June 9, 2018

accepted after revision

July 27, 2018
DOI https://doi.org/

10.1055/s-0038-1669931. ISSN 2475-4757.
Copyright $\odot 2018$ by Thieme Medical Publishers, Inc., 333 Seventh Avenue, New York, NY 10001, USA. Tel: +1(212) 584-4662.
License terms

(요 (1) $\Theta$ 
Ophthalmic surgical teaching has traditionally occurred by an apprenticeship model. However, simulation and stepbased procedural learning can allow for a more efficient, uniform, and comprehensive learning experience. ${ }^{1}$ Focusing on the individual steps of a surgery allows the learner to focus on achieving competency in increments and facilitates efficient teaching. Successful curricula for procedures such as cataract and strabismus surgery incorporate structured wet laboratories and simulations to improve resident preparedness as primary surgeons. ${ }^{2,3}$ Most ophthalmology programs employ surgical curricula wherein senior residents (postgraduate year 4 [PGY-4]) focus on intraocular surgery, especially cataract surgery, during their final year of training after having completed an intraocular surgery educational curriculum during the early stages of residency.

In recent years, microinvasive glaucoma surgery (MIGS) has gained prominence within ophthalmology, with many MIGS procedures designed for and intended to be performed concurrently with cataract surgery. The iStent (Glaukos Corporation, Laguna Hills, CA) is a trabecular microbypass stent for the reduction of intraocular pressure (IOP) in patients with mild to moderate open-angle glaucoma that is intended for placement at the time of cataract surgery. Given the increasing popularity and efficacy of MIGS procedures, and specifically the iStent trabecular microbypass stent, ${ }^{4,5}$ we sought to develop a graded curriculum to educate our residents in this technique. A surgical curriculum for microbypass stent placement has not previously been described.

In this study, we seek to determine whether a three-stage surgical curriculum for resident surgeons leads to successful insertion of a trabecular microbypass stent.

\section{Methods}

\section{Study Design}

This study was approved by the University of Chicago Institutional Review Board and followed the tenets of the Declaration of Helsinki. Senior (PGY-4) ophthalmology residents ( 9 residents) from three classes over 3 years (academic year 2015-2016, academic year 2016-2017, and academic year 2017-2018) participated in a curriculum to learn how to insert the iStent. The curriculum ( - Table 1 ) involved several distinct stages, with each stage requiring a resident to successfully complete the surgical tasks set forth in the curriculum under the supervision and approval of an experienced attending surgeon at all steps (K.M.R.).

Primary success was determined by correct intraoperative placement of the device confirmed via independent intraoperative gonioscopy by the supervising surgeon. Secondary success was measured by change in IOP and number of topical hypotensive medications used after surgery, determined through chart review. Patients included in the study were all patients that underwent cataract surgery with iStent placement performed by a resident as the primary surgeon between October 2015 and December 2017. There were 43 cases that fully met the criteria as outlined above.

Following the initiation of the study, former residents that participated in the curriculum were sent a link to a brief online survey (SurveyMonkey Inc, San Mateo, CA) via email. The survey had seven items and they were asked to rate their agreement on a 5 -point Likert scale ( $1=$ Strongly disagree, $2=$ Disagree, $3=$ Neutral, $4=$ Agree, $5=$ Strongly Agree). A free text box was available for individuals to elaborate upon their agreement ratings. Responses were anonymous. Topics included preparedness following the wet laboratory, effectiveness of the three-stage curriculum for preparing residents to perform the procedure during residency, preparedness after residency, and satisfaction with surgical results. A final freeresponse box offered the opportunity to suggest ways to improve the curriculum.

\section{The Curriculum}

\section{Phase 1}

A stepwise wet laboratory with synthetic materials and human cadaveric tissue was held early in the academic year so that residents could gain familiarity with the device and develop bimanual surgical dexterity (- Table 1; - Fig. 1A-D). Prior to the residents practicing tasks at the various wet laboratory stations, a representative from Glaukos reviewed the following key topics: indications for the iStent trabecular microbypass stent, visualization of the trabecular membrane with the gonioscope, angle anatomy, positioning the patient, mechanism of steps for insertion and technique, and procedural checks after insertion to make sure the stent is in place. Learners also discussed the advantages and disadvantages of performing stent placement before or after phacoemulsification. The representative reviewed the mechanism of action and design of the iStent and its inserter with a model that was 25 times the size of the real device. Following the presentation, residents practiced skills through a series of ordered tasks, moving from one to the next only after demonstrating competency at each skill station. Video displays and teaching microscopes allowed the representative and supervising surgeons to offer guidance and feedback.

First, residents used an eye model filled with viscoelastic to practice visualizing the trabecular meshwork with the gonioscope. Learners worked to improve bimanual dexterity by using a gonioscope in the nondominant hand and practicing the motions of maneuvering an empty inserter through an incision in the eye model toward the angle. Next, residents practiced inserting the stent on a synthetic foam pad (-Fig. 2A). The texture of the material was intended to simulate that of the trabecular meshwork. Residents practiced angling their hand and wrist, the angle of approach for the stent, keeping their hands steady, and relaxing the arm before releasing the stent from the inserter. Upon placement and "insertion" of the stent, learners practiced the procedural checks to ensure the stent is secure: using the inserter to tap the stent forward at the heel and "strumming" the nozzle vertically. After this exercise, residents practiced inserting and removing the stent into a three-dimensional printed artificial trabecular meshwork (-Fig. 2B). This model allows learners to practice insertion at various angles and against different levels of tension, preparing them for the anatomic and structural variation to be encountered on 
Table 1 Curriculum goals and objectives

\begin{tabular}{|c|c|c|}
\hline Phase & Goals & Objectives \\
\hline \multirow[t]{3}{*}{1 Wet laboratory } & $\begin{array}{l}\text { Understand indications for iStent, } \\
\text { design of device, and necessary } \\
\text { preoperative planning }\end{array}$ & $\begin{array}{l}\text { 1. Learner can list indication for the } \\
\text { iStent (and contraindications) } \\
\text { 2. Learner can name parts of the device, } \\
\text { describe where they should be placed } \\
\text { anatomically, and describe its } \\
\text { mechanism of action } \\
\text { 3. Learner can describe at least one } \\
\text { advantage and disadvantage for } \\
\text { performing the procedure before } \\
\text { phacoemulsification and after } \\
\text { phacoemulsification }\end{array}$ \\
\hline & $\begin{array}{l}\text { Improve skill of gonioscopy and } \\
\text { visualization of angle anatomy (iPrism, } \\
\text { Glaukos Corporation, Laguna Hills, CA) }\end{array}$ & $\begin{array}{l}\text { 4. Learner can name all key structures } \\
\text { and landmarks in angle anatomy } \\
\text { 5. Learner can describe the correct } \\
\text { location for insertion and would be } \\
\text { able to identify it on an image and } \\
\text { synthetic model }\end{array}$ \\
\hline & $\begin{array}{l}\text { Improve bimanual dexterity } \\
\text { Competency in following aspects of } \\
\text { insertion technique: holding the } \\
\text { inserter, angle of approach, insertion } \\
\text { into trabecular meshwork, release of } \\
\text { stent from inserter, strumming, and } \\
\text { tapping heel of stent to make sure it is } \\
\text { securely in place } \\
\text { Competency in using the inserter to } \\
\text { successfully regrasp and reposition the } \\
\text { iStent }\end{array}$ & $\begin{array}{l}\text { 6. Eye model: Learner maneuvers the } \\
\text { empty inserter into the anterior } \\
\text { chamber (AC) and toward the trabe- } \\
\text { cular membrane without losing } \\
\text { visualization with the gonioscope } \\
\text { 7. Synthetic pad: Learner inserts and } \\
\text { removes the iStent utilizing the } \\
\text { correct angle of approach } \\
\text { 8. Artificial trabecular meshwork: } \\
\text { resident successfully and correctly } \\
\text { (in terms of angle of approach, hand } \\
\text { position) inserts and removes the } \\
\text { device from different areas } \\
\text { 9. Human cadaveric tissue: Learner } \\
\text { successfully inserts the device into } \\
\text { cadaveric trabecular meshwork using } \\
\text { the surgical microscope. Learner also } \\
\text { practices regrasping and reposition- } \\
\text { ing to device to simulate surgical } \\
\text { adjustments that may be necessary in } \\
\text { live patient cases }\end{array}$ \\
\hline $\begin{array}{l}2 \text { Visualization of angle anatomy at the } \\
\text { conclusion of uncomplicated cataract } \\
\text { surgery (without insertion of iStent) }\end{array}$ & $\begin{array}{l}\text { Transfer of skills learned in wet labora- } \\
\text { tory to operating room environment } \\
\text { Improve visualization of the angle } \\
\text { anatomy through intraoperative } \\
\text { gonioscopy } \\
\text { Develop competency with intraopera- } \\
\text { tive adjustment of patient and } \\
\text { microscope position }\end{array}$ & $\begin{array}{l}\text { 1. Learner successfully rotates the } \\
\text { surgical microscope and adjusting } \\
\text { the patient's head to properly } \\
\text { visualize the nasal angle and } \\
\text { trabecular meshwork } \\
\text { 2. Learner successfully inserts the } \\
\text { viscoelastic cannula near the } \\
\text { trabecular meshwork during routine } \\
\text { phacoemulsification procedures, } \\
\text { without complications } \\
\text { 3. Learner can use the nondominant } \\
\text { hand to position the gonioprism and } \\
\text { dominant hand to manipulate the } \\
\text { viscoelastic cannula }\end{array}$ \\
\hline 3 iStent insertion & $\begin{array}{l}\text { iStent insertion with attending } \\
\text { supervision }\end{array}$ & $\begin{array}{l}\text { 1. Learner visualizes trabecular } \\
\text { meshwork and inserts iStent into } \\
\text { correct anatomical location, } \\
\text { performs procedural checks to make } \\
\text { sure the device is secure and } \\
\text { functioning } \\
\text { 2. Independent intraoperative } \\
\text { gonioscopy by supervising surgeon } \\
\text { confirms correct anatomical } \\
\text { placement of the iStent }\end{array}$ \\
\hline
\end{tabular}



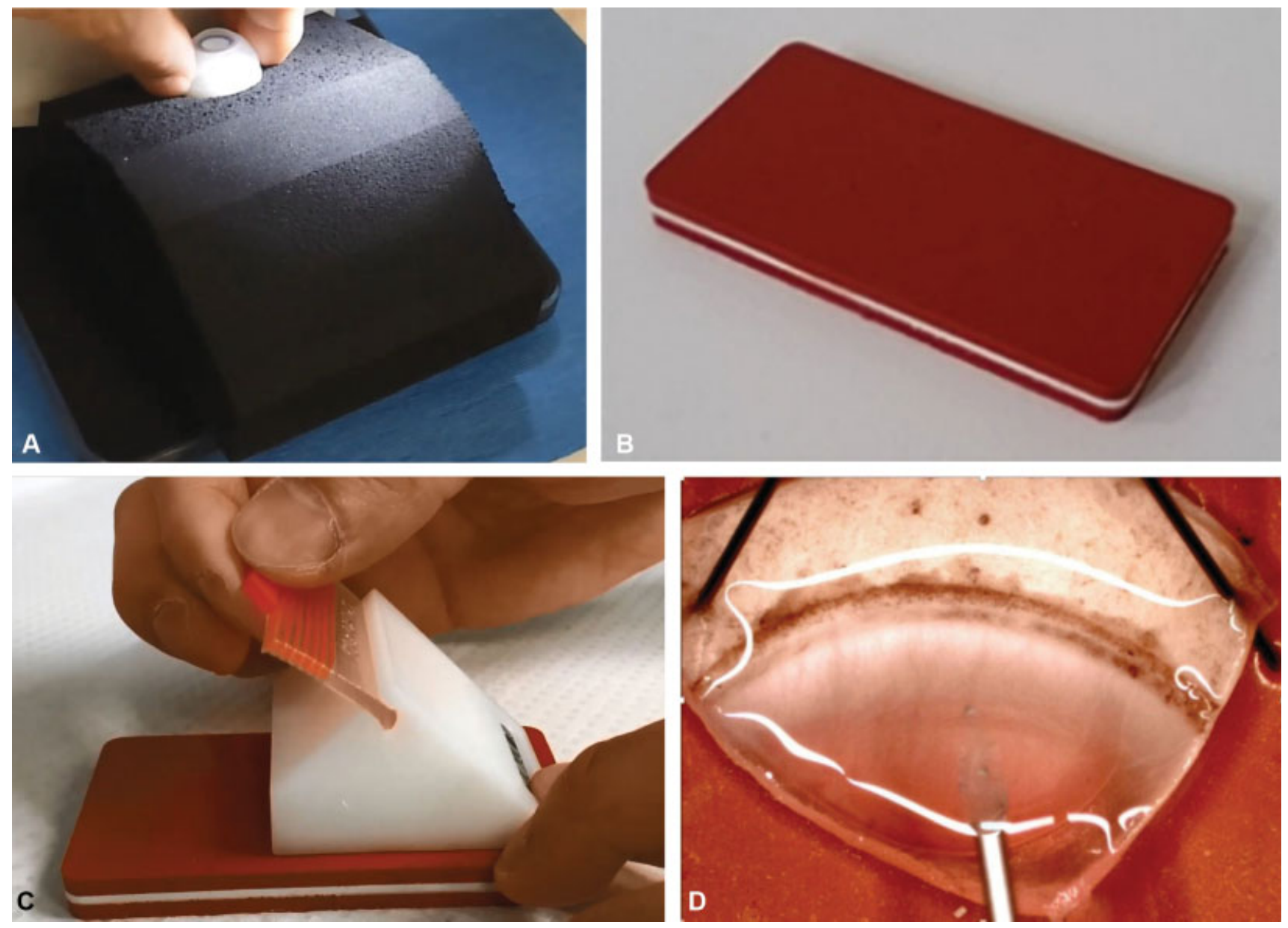

Fig. 1 (A) Eye model for practicing gonioscopy. (B) Synthetic pad mimicking texture of trabecular meshwork. (C) Artificial trabecular meshwork. (D) Human cadaveric tissue.
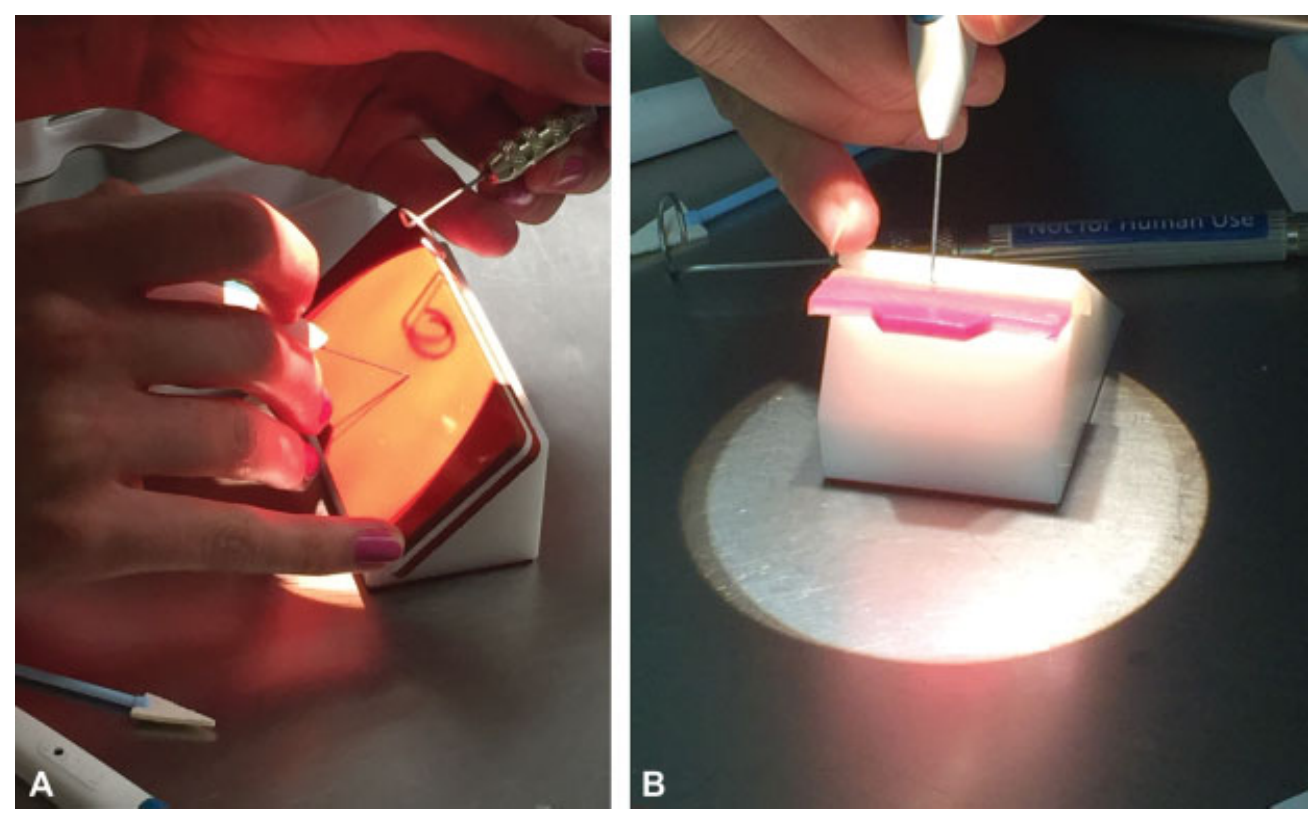

Fig. 2 (A) A resident practices iStent insertion with a synthetic foam pad. (B) A resident practices insertion into an artificial trabecular meshwork model. 
future patients. Finally, residents practiced placing the stent in the trabecular meshwork of human cadaveric tissue.

\section{Phase 2}

In the second phase of the curriculum, residents practiced visualizing angle anatomy with intraoperative gonioscopy at the conclusion of uncomplicated phacoemulsification cases. A significant component of this step was for a resident surgeon to develop familiarity with rotating the patient's head nasally and adjusting the surgical microscope to allow for a proper approach to visualize the angle. It was also an opportunity to practice adapting previously acquired skills to the clinical variation encountered with live patients. Once residents demonstrated proficiency with intraoperative gonioscopy, they were allowed to simulate placement of the iStent into the trabecular meshwork using an empty viscoelastic cannula. Residents typically performed 5 to 10 of these practice cases, with proficiency assessed by the same attending surgeon.

\section{Phase 3}

As primary surgeons, residents performed supervised insertion of the device in patients with mild-to-moderate primary open-angle glaucoma who met the criteria for stent placement at the time of cataract surgery. All resident procedures were supervised by the same attending surgeon who supervised the wet laboratory training (K.M.R.).

\section{Analysis}

Patients were identified in the electronic medical record (EMR) using date of birth (DOB) and last name. Preop IOP was an average of all IOPs recorded in the 12 months prior to surgery. Post-IOP and medication use at 1 day, 1 week, 1 month, 3 months, 6 months, and 12 months were manually extracted from the EMR. Operative reports and patient encounter notes were evaluated for complications. Thirteen patients (30\%) had not reached the 12 -month postop visit but had a 6-month follow-up visit. For seven cases (16\%), the patients missed the 6-month follow-up appointment but were present for the 12-month visit. All patients had at least one follow-up appointment at 6 or 12 months after the procedure. Data analysis was performed using Microsoft Excel (Microsoft Corporation, Redmond, WA) and Stata/SE 12 (StataCorp, College Station, TX). Shapiro-Wilk tests were performed on the IOP measurements at all time points to assess for normal distribution. A $t$-test for matched pairs was performed to compare samples determined to be normally distributed. Not all samples were determined to be normally distributed, and in these cases, a Wilcoxon sign-rank test was used to compare IOP at follow-up time points to preop IOP.

Studies were identified for comparison from PubMed using the following search words "iStent," "outcomes," "ophthalmology," "surgical education," and "microbypass stent."

\section{Results}

\section{iStent Insertion}

There were a total of 43 resident-performed cases between October 2015 and December 2017 that met the inclusion criteria. There were no significant intraoperative complications including loss of implant, iris or anterior segment trauma, or hyphema. The iStent insertion was performed after phacoemulsification in all cases. The iStent location was confirmed intraoperatively by the supervising attending surgeon and during postoperative visits via in-office gonioscopy and was noted to be in good position for the duration of the follow-up period. There were five cases $(12 \%)$ with rebound anterior chamber inflammation in the first postop month that resolved with treatment. One patient required an additional goniotomy procedure to lower IOP and another patient underwent trabeculectomy.

\section{Patient Outcomes: Intraocular Pressure and Medication Requirements}

Average preoperative IOP was $17.5 \pm 4.0 \mathrm{~mm} \mathrm{Hg}$. Six-month postop data were available for $36 / 43$ (84\%) cases and the average IOP was $14.5 \pm 2.2 \mathrm{~mm} \mathrm{Hg}$ or a $13 \%$ reduction from baseline ( $\pm 15 \%)$. Thirty out of 43 cases $(70 \%)$ reached a 12-month follow-up visit and the average IOP was $14.5 \pm 3.5 \mathrm{~mm} \mathrm{Hg}$, with a reduction of $14 \% \pm 18 \%$. When comparing postoperative IOP to baseline IOP, a statistically significant reduction in the mean was found between 6 months postop and baseline $(p=0.0002)$ and 12 months postop and baseline $(p=0.0001)$.

Mean preop number of medications was $1.4 \pm 1.0$, and the mean number of medications at follow-up was $1.2 \pm 1.1$. Combination medications were considered two medications. Twenty-three of the 43 cases (53\%) had no changes in medication requirements, while $16 / 43$ (37\%) had a decrease in medication requirements. Only 4 cases had an increase in medications.

\section{Resident Survey}

All 9 residents were surveyed with a response rate of $8 / 9$ (89\%). The results of the survey are summarized in - Fig. 3. All respondents strongly agreed or agreed that the wet laboratory prepared them to insert iStents on live patients. One person expressed that having more time in the wet laboratory would have been useful but was still satisfied with surgical results in residency. One individual disagreed that they had sufficient practice during phase 2 but still agreed that the curriculum adequately prepared them to perform the procedure. Nearly all respondents felt that the curriculum had prepared them to perform iStent insertion on live patients after their residency training. One trainee stated that they received additional training in fellowship, and another indicated that they have not performed the procedure after residency. No respondents strongly disagreed with any statements.

\section{Discussion}

In the setting of rapid innovation and development of surgical technology in ophthalmology, residency programs are challenged to teach their trainees the newest techniques. Acquiring the skills to perform new and innovative procedures prepares residents to provide the best care for their future patients, and 


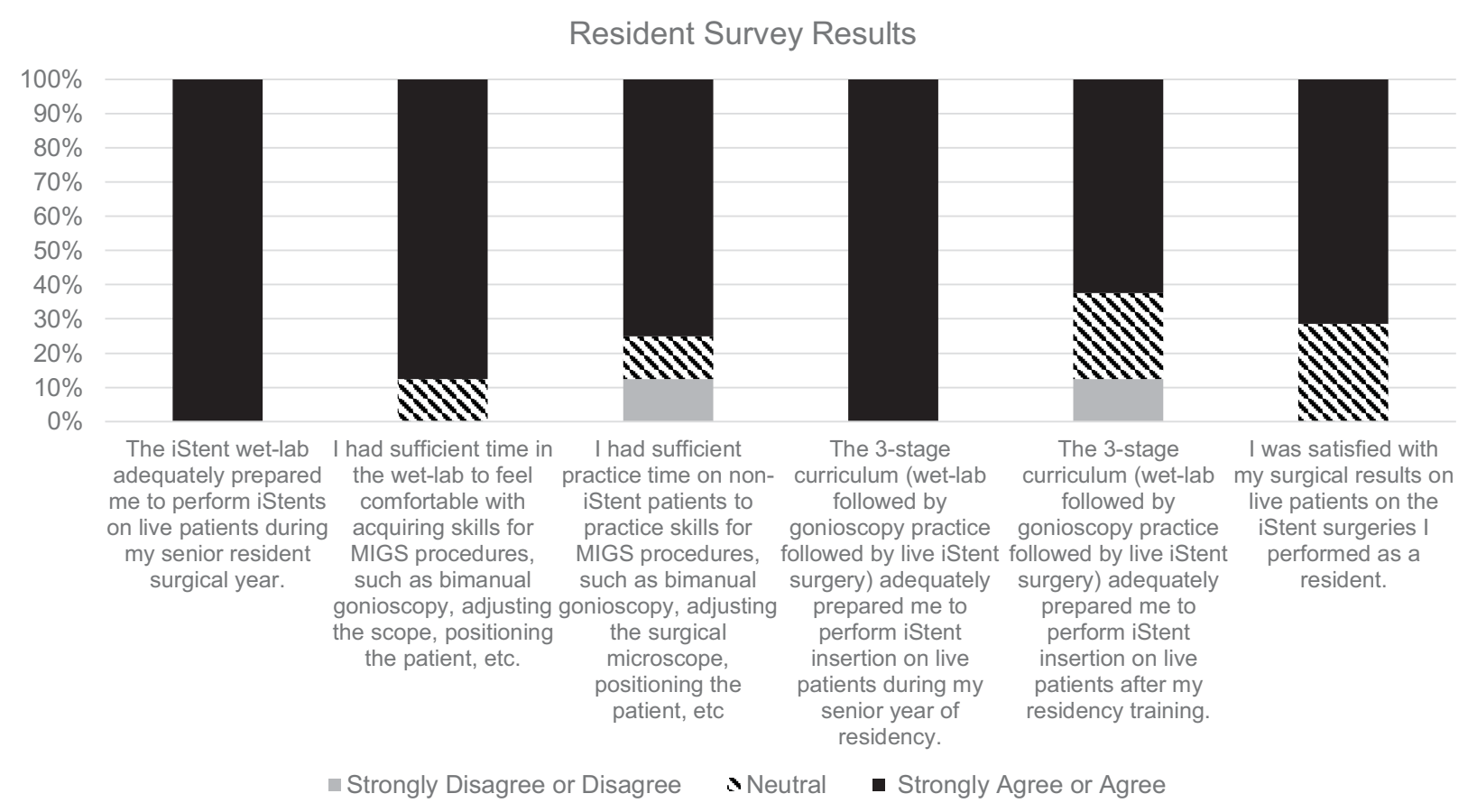

Fig. 3 Bar graph illustrating the proportion of respondents $(N=7)$ agreeing or disagreeing with statements on the curriculum follow-up survey. No respondents strongly disagreed with any statement.

also enables them to be competitive for employment opportunities after residency. MIGS procedures are a fitting example, given their efficacy and increasing popularity. The small size of microbypass stents and the unique skill-set for their insertion pose technical challenges for surgeons, even those with experience in microsurgical procedures and ophthalmic surgery. MIGS procedures require intraoperative manipulation of the microscope and appropriate rotation of the patient's head to afford the surgeon adequate view and access to the target tissue. Thus, visualization and placement of the iStent requires true bimanual dexterity and is well-suited to a stepwise mastery of skills. The distinct stages of our curriculum helped resident surgeons gain familiarity and confidence with the components of the procedure and enabled them to combine these skills to successfully perform the procedure. Our stepwise curriculum for microbypass stent placement is a model for incorporating the instruction of new skills into existing surgical training curricula.

The primary outcome we measured for the purpose of this study was for residents to successfully complete the surgical curriculum and perform iStent placement in patients. All residents met this primary measure without any intraoperative complications. When surveyed about the effectiveness of the three-stage curriculum, residents felt that it adequately prepared them to perform iStent insertion on live patients during residency and after residency. We will further improve the curriculum by continuing to seek feedback from future residency classes immediately after the completion of their senior year. Validated assessment tools such as an Ophthalmology Surgical Competency Assessment Rubric for microbypass stent placement are not currently available. The development of an assessment rubric in the future will be useful for evaluating and strengthening curricula dedicated to this procedure. As a secondary measure, we also assessed patient outcomes after resident-performed iStent implantation to determine the success of the curriculum and noted varying levels of success in this regard. In terms of IOP reduction, the patient outcomes were comparable to published results of surgeons' early cases of combined phacoemulsification and iStent insertion. ${ }^{6-10}$ This suggests that our residents' performance was not inferior to that of other surgeons new to the procedure. While the focus of this article is not the efficacy of the iStent, these patient outcomes support the promising results of our curriculum.

In summary, after participating in a three-stage surgical curriculum for trabecular microbypass stent placement, residents were able to perform the procedure without intraoperative complications and with minimal postoperative incidents, and to achieve significant reduction in IOP for their patients. Their success demonstrates that our graded curriculum may be a useful model for other residency programs desiring to train their residents in microbypass stent placement as well as other innovative surgical procedures.

\section{Conflict of Interest}

None.

\section{References}

1 Lorch AC, Kloek CE. An evidence-based approach to surgical teaching in ophthalmology. Surv Ophthalmol 2017;62(03):371-377

2 Kloek CE, Borboli-Gerogiannis S, Chang K, et al. A broadly applicable surgical teaching method: evaluation of a stepwise introduction to cataract surgery. J Surg Educ 2014;71(02):169-175 
3 Gertsch KR, Kitzmann A, Larson SA, et al. Description and validation of a structured simulation curriculum for strabismus surgery. J AAPOS 2015;19(01):3-5

4 Malvankar-Mehta MS, Iordanous Y, Chen YN, et al. iStent with phacoemulsification versus phacoemulsification alone for patients with glaucoma and cataract: a meta-analysis. PLoS One 2015;10(07):e0131770 Doi:10.1371/journal.pone.0131770

5 Neuhann TH. Trabecular micro-bypass stent implantation during small-incision cataract surgery for open-angle glaucoma or ocular hypertension: Long-term results. J Cataract Refract Surg 2015; 41(12):2664-2671

6 Fea AM. Phacoemulsification versus phacoemulsification with micro-bypass stent implantation in primary open-angle glaucoma: randomized double-masked clinical trial. J Cataract Refract Surg 2010;36(03):407-412
7 Spiegel D, García-Feijoó J, García-Sánchez J, Lamielle H. Coexistent primary open-angle glaucoma and cataract: preliminary analysis of treatment by cataract surgery and the iStent trabecular microbypass stent. Adv Ther 2008;25(05):453-464

8 El Wardani M, Bergin C, Achache F, Sharkawi E. Evaluating the trabecular micro-bypass stent combined with phacoemulsification compared to phacoemulsification alone. Klin Monatsbl Augenheilkd 2015;232(04):442-445

9 Arriola-Villalobos P, Martínez-de-la-Casa JM, Díaz-Valle D, et al. Mid-term evaluation of the new Glaukos iStent with phacoemulsification in coexistent open-angle glaucoma or ocular hypertension and cataract. Br J Ophthalmol 2013;97(10):1250-1255

10 Patel I, de Klerk TA, Au L. Manchester iStent study: early results from a prospective UK case series. Clin Experiment Ophthalmol 2013;41(07):648-652 